$52 \mid 2014$

Grammaire et enseignement du français langue

étrangère et seconde - Permanences et ruptures du XVIe au milieu du XXe siècle (II)

\title{
Les exercices de grammaire de Grevisse.Analyse des Exercices sur la grammaire française de M. Grevisse, 1942
}

\section{Rosa Leandra Badalamenti}

\section{OpenEdition}

\section{Journals}

\section{Édition électronique}

URL : https://journals.openedition.org/dhfles/3623

DOI : $10.4000 /$ dhfles.3623

ISSN : 2221-4038

\section{Éditeur}

Société Internationale pour l'Histoire du Français Langue Étrangère ou Seconde

\section{Édition imprimée}

Date de publication : 1 juin 2014

Pagination : 165-180

ISSN : 0992-7654

Référence électronique

Rosa Leandra Badalamenti, « Les exercices de grammaire de Grevisse.Analyse des Exercices sur la grammaire française de M. Grevisse, 1942 ", Documents pour l'histoire du français langue étrangère ou seconde [En ligne], 52 | 2014, mis en ligne le 06 août 2016, consulté le 28 mai 2021. URL : http:// journals.openedition.org/dhfles/3623 ; DOI : https://doi.org/10.4000/dhfles.3623

Ce document a été généré automatiquement le 28 mai 2021

(c) SIHFLES 


\section{Les exercices de grammaire de Grevisse.Analyse des Exercices sur la grammaire française de M. Grevisse, 1942}

Rosa Leandra Badalamenti

\section{Introduction}

1 Les Exercices sur la grammaire française sont parus pour la première fois en 1942, à Gembloux, pour les éditions Duculot. Le texte était adapté au Précis de grammaire française, manuel scolaire publié par Grevisse en 1939, trois ans après Le Bon Usage, qui allait connaître un succès retentissant auprès de générations d'élèves du français langue maternelle mais aussi $d u$ français langue étrangère. Dans le contexte universitaire surtout, le Précis de Grevisse a été l'un des ouvrages de référence pour des générations d'étudiants étrangers. Les Exercices, pour leur part, ont été souvent utilisés par les professeurs de français langue étrangère pour des activités de classe ou pour des examens ${ }^{1}$.

2 Bien sûr, l'œuvre de Grevisse était destinée à un public d'étudiants de français langue maternelle et les Exercices ne constituent pas une exception. Il est cependant intéressant de noter que, en parallèle de la diffusion des méthodes béhavioristes dans l'enseignement $d u$ français langue étrangère à partir des années '60, un nombre important d'enseignants italiens étaient encore liés à des méthodes grammairetraduction, et même à des ouvrages destinés à un public français.

3 L'œuvre de Grevisse s'insère dans le sillon de la grammaire traditionnelle, telle qu'elle a pris forme depuis le XVIII ${ }^{\mathrm{e}}$ siècle avec la grammaire de Lhomond (Bourdon 2008). Selon Swiggers (1987 : 104), Le Bon Usage - mais on peut aisément en dire de même du Précis constitue le « rejeton d'un type de grammaire qui a son origine dans le XVII ${ }^{\mathrm{e}}$ siècle, et qui vise à mettre en rapport un système de marques formelles avec un ensemble de 
contenus sémantiques ». D'où le développement d'une théorie grammaticale établissant des catégories de forme et de fonctions figées en paradigmes. C'est donc à partir d'une telle conception de la grammaire, comme système de marques sémantico-formelles et de règles, que prennent corps les exercices de grammaire de Grevisse. En outre, comme le remarque Wilmet (1997), la grammaire de Grevisse s'inspire d'un «bon usage », c'està-dire de la pratique des «bons » écrivains, ce qui en fait une grammaire strictement normative.

4 En ce qui concerne les Exercices, il semble clair que ceux-ci reflètent en quelque sorte les théories grammaticales de référence, tout en procédant par ailleurs d'une série de facteurs différents, comme la conception de la norme linguistique, les modèles scolaires de référence, les objectifs de l'enseignement, etc. (Bastuji 1977). Les premiers exercices de grammaire font leur apparition, dans les manuels scolaires de langue étrangère, au XIX ${ }^{e}$ siècle (Minerva \& Pellandra 1997). Selon Besse et Porquier (1991), bien qu'il n'existe aucune étude systématique portant sur l'évolution des exercices scolaires de grammaire, la structure de ceux-ci demeure inchangée par rapport à l'évolution des théories linguistiques. Les exercices de grammaire constituent un outil pédagogique bien ancré dans la tradition scolaire et justifié par les nécessités de l'alphabétisation ou de l'amélioration de la langue écrite (Bastuji 1977). Pour ces raisons, la pratique de l'exercice de grammaire en classe de français langue étrangère conserve son rôle central, surtout en contexte institutionnel. S'approcher d'un texte comme Exercices sur la grammaire française et en analyser les tâches métalinguistiques nous permet de mieux comprendre la conception et le rôle de la grammaire dans la première moitié du $\mathrm{XX}^{\mathrm{e}}$ siècle. D'ailleurs, celle-ci a influencé non seulement l'enseignement de la langue maternelle, mais aussi l'enseignement/apprentissage des langues étrangères.

\section{Exercices sur la grammaire française. Structure et distribution des exercices}

5 Il faut tout d'abord souligner que les Exercices sont conçus comme un instrument de et pour la classe de français, et qu'ils sont donc inscrits dans un "rituel communicatif » (Besse \& Porquier 1991: 120) propre à la classe de langue. L'exercice est donc une activité gérée par l'enseignant, à laquelle les apprenants répondent selon les consignes, et après laquelle l'enseignant fournit un feedback. Il s'agit donc d'un échange ternaire (Sinclair \& Coulthard 1975) dont la correction par l'enseignant constitue la dernière étape. D'ailleurs, le texte est accompagné d'un Livre du maitre avec les solutions des exercices proposés. Les apprenants n'ont donc accès au feedback que par le biais de la correction fournie par l'enseignant.

6 Les treize chapitres sont ainsi organisés : le premier traite, de manière générale, des éléments de la langue (les sons, les signes, les mots); le second est consacré à la structure de la proposition avec ses éléments (le sujet, les compléments, etc. pour finir avec les types de propositions); les chapitres III-XI sont consacrés aux parties du discours; le chapitre XII traite des propositions subordonnées et le chapitre XIII de la ponctuation. L'organisation des exercices se fait en fonction de la répartition de la grammaire en phonétique, morphologie et syntaxe. Toute la partie centrale du texte (chapitres III-XI) s'organise autour de la répartition traditionnelle des parties du discours. Il s'agit donc d'exercices métalinguistiques, où les activités requises par l'enseignant portent sur un ou quelques aspects de grammaire spécifiques, avec pour 
but, de la part des apprenants, de fixer, par réitération de la tâche, les modalités d'accomplissement de celle-ci. Dans cette perspective il est intéressant de remarquer que les exercices de récapitulation sont rares et ne concernent que les chapitres consacrés au nom (et notamment la formation du féminin et du pluriel des noms), aux adjectifs (typologie et accord de l'adjectif), aux verbes et aux propositions subordonnées.

7 Le texte comprend 758 exercices de grammaire, distribués de manière différente selon le point de grammaire traité. Dans le premier chapitre on compte 84 exercices, dont 19 exercices oraux concernant la phonétique (distinction de consonnes, voyelles ou syllabes et liaison). Des 84 exercices, 65 sont consacrés aux mots et comprennent des exercices portant sur l'origine des mots ( $c f . \S 3.3$, ex. 1), sur les procédés de dérivation et de composition et sur les relations sémantiques d'homonymie, synonymie, antonymie et paronymie. Le deuxième chapitre, consacré à la proposition, comprend 88 exercices distribués selon les catégories traditionnelles dans l'analyse de la phrase simple. En ce qui concerne les chapitres consacrés aux parties du discours (III-XI), les exercices sont distribués de manière irrégulière avec un noyau dur de 212 exercices concernant le verbe. Le chapitre dédié aux propositions subordonnées comprend 121 exercices répartis selon les catégories traditionnelles de l'analyse de la phrase complexe. On relève encore 46 exercices pour le nom, 20 pour l'article, 87 pour l'adjectif, 35 pour le pronom, 28 pour l'adverbe, 13 pour la préposition, 13 pour la conjonction et 2 pour l'interjection. Pour finir, le douzième chapitre, sur la ponctuation, comprend 8 exercices.

\section{Les classements des exercices de grammaire}

8 L'inertie relative des exercices grammaticaux a permis de dresser des classements assez réguliers. À partir des typologies proposées par Bastuji relativement au travail demandé aux étudiants de français langue maternelle² (Bastuji 1977 : 9-10), Besse et Porquier (1991: 124) regroupent les exercices de grammaire en quatre grandes catégories : l'exercice de répétition, l'exercice à trous, l'exercice structurel et l'exercice de reformulation. Réquédat (1968) et Delattre (1971) dressent une typologie des exercices structuraux typiques des méthodes audio-orales liées au béhaviorisme. Delattre met l'accent sur les trois dimensions qui interviennent dans la classification des exercices structuraux: la nature des relations sur lesquelles l'exercice s'appuie (similarité ou opposition), les mécanismes de base impliqués dans l'accomplissement des tâches (répétition, substitution, transformation), le niveau linguistique concerné par l'exercice (phonétique, grammatical, lexical). Il y a bien sûr une différence substantielle entre ceux-ci et les exercices traditionnels : ces derniers se basent sur des explicitations métalinguistiques, tandis que les exercices structuraux ont pour but l'acquisition d'un automatisme et relèvent donc, dans les intentions de ces théoriciens (Besse et Porquier 1991), de la grammaire implicite. Cependant, la structure des exercices ne change pas de manière radicale : les exercices visant à une compétence implicite partent d'une structure-modèle ou pattern (Réquédat, 1968) à partir duquel l'apprenant automatise, par répétition, la structure envisagée, tandis que les exercices traditionnels visant à une compétence explicite présentent des consignes métalinguistiques à partir desquelles l'apprenant effectue la tâche requise par l'exercice avec pour but l'acquisition de règles explicites de grammaire. En ce qui 
concerne les exercices traditionnels, il faut citer aussi le classement proposé par Cristea (1984), qui identifie sept grands types d'exercices : les exercices d'analyse, les exercices de maniement de paradigme, les exercices de restitution, les exercices de combinaison, les exercices d'adjonction d'éléments, les exercices d'invention et les exercices mixtes.

Pour ce qui concerne les Exercices sur la grammaire française, nous avons pu les classer, d'un point de vue formel, selon les typologies suivantes:

1. Exercices de distinction, repérage, classement et analyse

2. Exercices de répétition ou réitération

3. Exercices d'appariement

4. Exercices d'expansion

5. Exercices de transformation et de reformulation

6. Exercices à trous

10 Presque la moitié des exercices contenus dans le texte (47\%) peut être classée sous la première typologie (exercices d'analyse). Suivent des exercices de répétition (17\%) et d'expansion de phrase (19\%), des exercices de reformulation $(9 \%)$, des exercices à trous $(10 \%)$ et, pour finir, quelques exemples d'exercices d'appariement (1\%).

\subsection{Exercices de distinction, repérage, classement et analyse}

11 Dans la plupart des cas, les tâches consistent à distinguer des classes ou des fonctions dans des phrases ou bien à en justifier l'emploi. Dans d'autres exercices l'apprenant doit ranger les éléments d'une liste selon des critères donnés.

La plus grande partie des exercices d'analyse est comprise dans les chapitres I, II, VII et XII du livre, à savoir "Les éléments de la langue », «La proposition ", «Le verbe » et "Les propositions subordonnées », mais on en trouve aussi maints exemples dans les autres chapitres. Il s'agit de la classe d'exercices la plus fournie et dont l'emploi est le plus systématique.

13 Comme pour les exercices d'analyse de Cristea (1984), l'objectif de ces exercices est le développement et l'évaluation des connaissances relatives au système de la langue. Les exercices requièrent de la part des apprenants une bonne connaissance du métalangage grammatical, comme le montrent les exemples suivants :

1. 111. - Discernez les compléments circonstanciels de lieu et dites de chacun d'eux s'il marque : $1^{\circ}$ la situation ; $2^{\circ}$ la direction ; $3^{\circ}$ l'origine ; $4^{\circ}$ le passage. [Gr. §§ 55-56]

a) 1. Un pinson chante dans les feuillages. --- 2. Les Romains tiraient de la Sicile beaucoup de blé. --- 3. Le renard et le bouc descendirent dans un puits. --- 4. Nous goûterons au ciel un bonheur parfait. --- [...]

b) 1. Des hirondelles ont fait leur nid à l'angle de ma fenêtre. --- 2. De la forêt arrivent des effluves pénétrants. --- 3. Un agneau se désaltérait dans un clair ruisseau. --- [...]

(Exercices sur la grammaire française, $1942: 41-42$ )

2. 324. - Discernez les divers adjectifs : numéraux, possessifs, démonstratifs, relatifs, interrogatifs, indéfinis ; analysez-les : [Gr. \$§ 199-225]

1. Votre avenir, mes chers enfants, pour un bonne part du moins, dépend de vousmêmes. --- 2. Mille cris joyeux résonnent; divers murmures circulent dans les branches : toute cette agitation annonce le lever du jour. --- 3. Quels succès pouvezvous espérer si vous remplissez vos journées de je ne sais quelles frivolités ? --- [...]

(Ibid. : 117-118) 
Comme on le voit dans ces exemples, l'apprenant doit connaître les notions métalinguistiques auxquelles renvoient les consignes et, d'une fois à l'autre, définir une classe d'unités linguistiques (dire la catégorie à laquelle appartiennent certains mots, repérer les adjectifs et les classer selon leur typologie, discerner les verbes transitifs directs et les transitifs indirects, etc.), identifier et définir des mécanismes grammaticaux ou lexicaux (de l'interrogation directe à l'interrogation indirecte, la comparaison, la pronominalisation, ou encore la dérivation, etc.), reconduire les usages aux règles grammaticales qui les déterminent (justifier l'accord du participe ou des adjectifs, ou l'emploi de pronoms indéfinis, etc.) ou encore identifier, à partir d'une forme fléchie, le paradigme des unités en question.

\subsection{Exercices de répétition}

Sous cette classe, qui comprend $17 \%$ des exercices, nous avons fait entrer tous les exercices dont la tâche consistait à réitérer une forme, toujours donnée dans la consigne, sans aucune relation avec d'autres éléments de la phrase. Voici quelques exemples :

1. 183.--- Donnez le féminin de chacun des noms suivants : [Gr. § 86-93]

\begin{tabular}{|l|l|l|l|}
\hline chameau & espion & avocat & cousin \\
paysan & fou & Anglican & jumeau \\
berger & sot & candidat & Hottentot \\
sultan & faisan & prisonnier & écolier \\
Lapon & Gabriel & Persan & jouvenceau \\
Manchot & Grec & dévot & Wallon [...] \\
\hline
\end{tabular}

(Ibid. : 63)

2. 446. Mettez les verbes suivants à la forme indiquée :

[Gr. § 349 : geindre-paitre]

a) A la $1^{r e}$ pers. du futur simple: 1. Geindre. --- 2. Inclure. --- 3. Méconnaître. --- 4.

Oindre. --- 5. Paître. --- 6. Moudre. ---

b) A la $1^{r e}$ pers. du passé simple: 1 . Nuire. --- 2. Interdire. --- 3. Maintenir. --- 4. Luire.

--- 5. Lire. --- 6. Se méprendre. --- [...]

16 Les parties du discours concernées par ce type d'exercices sont le nom, l'adjectif et le verbe. Comme on le voit dans les exemples proposés, les exercices consistent à donner le féminin et le pluriel des noms et des adjectifs ou à conjuguer des verbes à des formes données. Bien que restreints à trois chapitres sur les treize que comporte le livre, ces exercices sont très nombreux.

17 Cristea (1984) appelle ce type d'exercices "exercices de maniement de paradigme ", insistant sur la nécessité de mémoriser tout le paradigme pour les accomplir. Le but de ces exercices est donc de faciliter ou d'évaluer la mémorisation des paradigmes de verbes, noms et adjectifs. 


\subsection{Exercices d'appariement}

\section{texte. Il s'agit de rapprocher des mots qui partagent le même radical ou qui} entretiennent des relations sémantiques de synonymie, antonymie ou paronymie.

19 1. 21. Dans les deux premières colonnes, rapprochez les doublets; de même, dans les deux dernières :

[Gr. § 24]

21 Modèle, boule --- bulle (Ibid. : 9)

\begin{tabular}{l|} 
boule \\
employer \\
veille \\
raide \\
examen \\
hôpital \\
trahison \\
serment \\
délicat \\
tempérer \\
picador \\
ouvrer \\
frêle
\end{tabular}

opérer
essaim
tremper
délié
tradition
vigile
rigide
bulle
hôtel
fragile
sacrement
impliquer
piqueur

dîme
natal
troubadour
chenal
chanteur
mansion
aigre
colloque
avoué
parabole
métier
cavalier
solide

Comme on le voit dans l'exemple proposé, la tâche consiste à rapprocher des noms qui partagent la même étymologie. Dans d'autres cas, les mots proposés doivent être appariés selon des critères sémantiques. Il s'agit des seuls exemples de sémantique lexicale dans le texte.

\subsection{Exercices d'expansion}

Ces exercices constituent presque $20 \%$ du nombre total et leur distribution est systématique : pour chaque chapitre il y a des exercices d'expansion dans lesquels la tâche consiste à former des phrases à partir d'un élément donné. Dans le premier chapitre, "Les éléments de la langue ", l'expansion s'opère à partir de mots par composition ou dérivation ( $c f$. $\$ 3.4$ ex. 1).

Par la suite, dans le chapitre sur la proposition il s'agit plutôt de former des phrases à partir d'une fonction, avec une attention particulière à la valence verbale, tout comme dans le chapitre 12, sur les propositions subordonnées, où l'expansion se fait à partir d'une proposition à compléter en ajoutant des subordonnées ou d'autres propositions. Nous proposons ici trois exemples d'exercices d'expansion :

1. 56. --- Au moyen du préfixe re- (ré-, res-, $r$-) formez des verbes composés des mots suivants et faites-les entrer chacun dans une expression : [Gr. \$ 30] Modèle : emmancher - remmancher une hache. 


\begin{tabular}{|l|l|l|l|}
\hline emmancher & boiser & serrer & servir \\
monter & partir & écurer & acheter \\
abaisser & abattre & suer & armer \\
cuire & produire & accommoder & sentir \\
agir & battre & montrer & emplir \\
appeler & pêcher & saisir & souder \\
toucher & unir & former & organiser \\
\hline
\end{tabular}

2. 112. Joignez à chacune des propositions suivantes un complément circonstanciel, selon l'indication mise entre crochets : [Gr. § 55-56]

1. Le corbeau tenait [lieu] un fromage. --- 2. Un coup affamé vint [lieu] observer l'état du troupeau. Il faut manger [but]. --- 4. La grenouille voulait égaler le bœuf [point de vue]. --- 5. Il ne faut pas faire l'aumône [cause]. --- 6. Le maître encourage l'élève [moyen]. --- 7. Le vaniteux parle constamment [propos]. --- 8. La forêt nous charme particulièrement [temps]. [...]

(Ibid. : 42)

3. 696. Joignez à chacune des propositions suivantes une subordonnée complément circonstanciel de comparaison :

[Gr. § 479]

1. De petits efforts répétés viennent à bout des plus grandes difficultés. --- 2 . L'homme fait sa vie. --- 3. Moins on éprouve de joies. --- 4. Il ne faut pas faire plus de promesses. --- 5. Rarement l'avenir est tel. --- 6. Vous comprendrez mieux la vie. --7. Après la pluie vient le beau temps. --- 8. La difficulté s'aplanira d'autant plus facilement. [...] (Ibid. : 274)

Ces exercices se basent sur l'expansion, à partir tout d'abord des unités lexicales, pour passer par la suite à l'expansion de la phrase simple et de la phrase complexe. Cristea (1984) identifie une classe dite d'exercices "d'invention ", visant au réinvestissement des éléments lexicaux ou grammaticaux acquis. Les élèves doivent former des mots dérivés ou composés à partir d'un nom, ou des phrases avec un ou plusieurs verbes, noms et adjectifs à une certaine forme, ou encore étendre une phrase simple ou complexe avec des compléments ou des propositions données, à partir donc de la fonction logique et sur des schémas donnés. Cependant, la partie laissée à la création, ou à l'invention selon la définition de Cristea, reste limitée et toujours liée à des structures préalablement données.

\subsection{Exercices de transformation et de reformulation}

Dans ces exercices ( $9 \%$ du total), la tâche consiste à restructurer des phrases ou à en changer les tournures. Ils sont bien sûr plus nombreux dans la partie relative au verbe. C'est le cas de la forme passive et des propositions subordonnées. En voici quelques exemples :

1. 165. --- Les propositions suivantes ont l'une des quatre formes: affirmative, négative, interrogative, exclamative. Donnez à chacune d'elles successivement les trois formes. (L'idée doit rester la même, mais certains mots peuvent, au besoin, être changés) :

[Gr. \$ 73] 
1. Notre âme est immortelle. --- 2. Les richesses ne font pas le bonheur. --- 3. Qu'il est beau, le pays natal! --- 4. Le printemps répand partout la joie. --- 5. Nos bonheurs ne durent guère. (Ibid. : 274)

\section{745. --- Transformez la construction des phrases}

[Gr. § 492] suivantes par l'emploi du style indirect libre :

1. Une femme se présenta et raconta que le malheur l'avait frappée, que son mari était malade, que ses enfants manquaient de vêtements, que son loyer n'était pas payé, qu'elle était absolument sans ressources. --- 2. L'hiver venu, la cigale alla trouver la fourmi et dit qu'elle souffrait cruellement de la faim, qu'elle suppliait sa charitable voisine de lui prêter quelques grains, qu'elle la payerait sans faute avant l'août. [...]

(Ibid. : 295)

Les exercices de transformation et de reformulation de phrases sont classés par Cristea (1984) parmi les exercices de restitution. Ils consistent à transformer une unité constitutive de la phrase en une autre unité avec laquelle elle entretient des rapports sémantiques constants, comme on le voit dans le premier exemple. Comme le remarquent Besse et Porquier (1991), ils sont exploités dans le cadre des modèles métalinguistiques liés aux théories de l'énonciation et de la pragmatique, comme dans le cas du discours rapporté ou des actes de parole. Dans notre cas, cependant, le but de l'exercice n'est pas de développer une compétence pragmatique ou en quelque sorte liée au contexte de l'énonciation; il s'agit plutôt d'automatiser des mécanismes grammaticaux par lesquels l'apprenant doit restituer une structure sous une autre forme. Par exemple, dans l'exemple proposé relatif au discours rapporté, il s'agit de supprimer la subordination donnant lieu au discours indirect pour restituer un discours indirect libre qui requiert des structures grammaticales différentes. L'accent n'est pas mis sur les stratégies narratives relevant du discours indirect libre ou sur les effets que celui-ci entraîne, mais sur la structure à maitriser. D'autres exercices, concernant la mise en relief ou la forme passive, répondent à la même exigence de manipulation de mécanismes grammaticaux.

\subsection{Exercices à trous}

Les exercices à trous (10\% du total) consistent à compléter des phrases en choisissant entre les éléments d'une liste ou d'une classe données. Ils ne sont pas nombreux, mais ils ont quand même une distribution régulière sur les treize chapitres du livre, et ils touchent à presque tous les sujets.

\section{314. Remplacez les points par le mot tout, que vous orthographierez correctement : [Gr. \$ 223]}

a) 1 .... les hommes doivent s'entraider comme des frères. --- 2. Il y a des phrases ... faites dont on se sert ... les jours sans en penser le sens. --- 3. ... Liège accueillit avec enthousiasme les libérateurs. --- 4. Nous ne savons le ... de rien : nos connaissances sont ... bornées. [...]

b) 1. Nos idées morales sont le reflet de notre vie ... entière. --- 2. Un homme qui a vécu dans l'intrigue pendant ... une longue période ne peut plus s'en passer : ... autre vie pour lui est fade et languissante. [...]

(Ibid. : 113)

2. 231. Remplacez les points par du, de la, de l', des --- ou par le simple de :

a) 1. Quand on a ... bonne volonté, on se concilie la bienveillance de ses chefs. --- 2 . Heureux les enfants qui ont ... grands-pères encore assez alertes pour les 
accompagner dans leurs promenades! Heureux aussi les grands-pères qui ont ... petits-enfants dociles et affectueux! --- 3. La paix du cœur procure ... douces jouissances. [...]

b) 1 . On a vu ... grands hommes mourir pauvres et ignorés. --- 2. Il est ... jeunes gens qui croient trouver ... vrais amis dans certains personnages qui ne sont que ... joyeux convives ou ... habiles flatteurs. [...]

(Ibid. : 295) restitution. Dans le cadre de la classification que nous avons adoptée, ils constituent une classe distincte en ce qu'ils requièrent la prise en considération de rapports syntagmatiques entre les éléments en présence et l'élément à insérer dans la phrase. L'apprenant doit, en plus d'avoir une bonne connaissance du paradigme de l'unité en question, évaluer les relations que celle-ci entretient avec les autres éléments de la phrase.

\section{Conclusions}

Bien que différents dans la forme et dans la distribution, les Exercices de Grevisse ont pour but la maîtrise d'un système métalinguistique. Plus en détail, les finalités des exercices peuvent être ainsi énumérées :

\section{Le stockage des informations en mémoire}

Tous les exercices analysés ont bien sûr comme premier objectif et effet de faciliter la mémorisation des formes. L'activité mnésique est fondamentale dans l'apprentissage traditionnel des langues. Dans certains cas, et nous renvoyons notamment à la section relative aux mots, l'accent est mis sur la création de réseaux sémantiques entre les mots, traduisibles en relations d'homonymie et paronymie, de synonymie et d'antonymie. Ces procédés sont d'ailleurs un soutien important à l'acquisition du lexique. Dans le même but, c'est-à-dire la mémorisation et l'automatisation des compétences métalinguistiques acquises, s'inscrivent les exercices de réitération ou de répétition de formes, de paradigmes, surtout irréguliers, et de classes de mots.

La catégorisation, c'est-à-dire la capacité de regrouper des éléments linguistiques selon des critères pertinents, demeure l'une des finalités les plus importantes des Exercices de Grevisse. Pour catégoriser, l'apprenant doit être en mesure de distinguer les éléments les uns des autres, de les repérer et de les classer selon leurs caractéristiques sémantiques, morphologiques ou syntaxiques. D'ailleurs, les exercices de distinction, repérage, classement et analyse constituent presque la moitié du corpus et témoignent donc de l'importance que revêt l'analyse de la structure dans les exercices traditionnels.

3. La déduction/induction des règles

Certains exercices visent à développer chez l'apprenant la capacité de comprendre ou dégager des règles de combinaison entre les éléments de la langue et la capacité de les appliquer quand les conditions nécessaires se présentent. C'est ici que se situe le passage entre les règles grammaticales fournies et l'exécution de la tâche langagière. Les opérations de déduction ou d'induction permettent aux apprenants de comprendre les dynamiques combinatoires entre les différents éléments de la langue et de les 
appliquer dans les exercices. Dans la plupart des cas, il s'agit de justifier l'emploi d'une forme à travers les règles de grammaire étudiées. " une tâche langagière ponctuelle à caractères répétitif, contraint et métalinguistique marqués, tâche demandée par le professeur aux étudiants et évaluée par lui » (1991 : 121). L'exercice de grammaire traditionnel répond aux objectifs fondamentaux et particuliers de la méthode traditionnelle : apprendre le vocabulaire de la langue et ses règles de grammaire (avec leurs exceptions). Les différents types d'exercices constituent donc la troisième étape de la démarche propre aux méthodes grammairetraduction : l'application et l'évaluation des connaissances théoriques acquises, après l'observation d'exemples et la définition de règles.

L'exercice de type traditionnel, sous ses différentes formes, constitue donc une étape orientée vers l'acquisition d'un outil métalinguistique, dans un parcours qui vise à la conceptualisation d'un système linguistique autonome par rapport à l'usage de la langue.

\section{BIBLIOGRAPHIE}

BASTUJI, Jacqueline (1977). « Pourquoi des exercices de grammaire ? ». Langue française, 33, 6-21. BESSE, Henry \& PORQUIER, Rémy (1991) [1984]. Grammaires et didactique des langues. Paris : HatierCrédif.

BOURDON, Marie-Claude (2008). « Après la nouvelle orthographe, la nouvelle grammaire ». Journal L'U.Q.A.M., XXXIV, 10, 2. 
CRISTEA, Teodora (1984). Linguistique et techniques d'enseignement. Bucarest : Universitatea din Bucureşti.

DELATTRE, Pierre (éd.) (1971). Les exercices structuraux pour quoi faire ? Paris : Hachette.

MINERVA, Nadia \& PELLANDRA, Carla (1997). Insegnare il francese in Italia. Repertorio analitico di manuali pubblicati dal 1625 al 1860. Bologna : Clueb.

PUREN, Christian (1988). Histoire des méthodologies de l'enseignement des langues. Paris : Nathan CLE International.

RÉQUÉDAT, François (1968). Les exercices structuraux. Paris : Hachette-Larousse.

SINCLAIR, John McHardy \& COULTHARD, Malcolm (1975). Towards an Analysis of Discourse. Oxford : Oxford University Press.

SWIGGERS, Pierre (1987). « Le Bon Usage de M. Grevisse : examen méthodologique et descriptif ». Revue Romane, Bind 22, 104-112.

WILMET, Marc (1997). Grammaire critique du français. Paris-Bruxelles : Hachette SupérieurDuculot.

\section{NOTES}

1. Nous avons proposé un questionnaire informatif à 11 anciens professeurs de français langue étrangère en service depuis la seconde moitié des années '60 jusqu'aux années '90. Tous les informateurs ont déclaré avoir utilisé régulièrement tant Le Bon Usage que le Précis pendant leurs activités de classe. Cinq d'entre eux ont déclaré avoir utilisé les Exercices pour des activités de classe et/ou pour des examens.

2. Le classement des exercices ne tient pas compte des activités de la classe de français, mais se limite à prendre en considération la structure de l'exercice (le travail demandé aux apprenants) avec ses consignes, tel qu'il est proposé aux apprenants.

\section{RÉSUMÉS}

Dans cette étude, nous analyserons les exercices de grammaire contenus dans Exercices sur la grammaire française de M. Grevisse, publiés à Gembloux en 1942 par les éditions Duculot. Nous examinerons les exercices présents dans le volume et détaillerons les différentes typologies d'exercices (d'analyse, d'identification, de transformation, de création, etc.), les finalités des différentes typologies d'exercices (procédures d'analyse et/ou de restructuration du système linguistique, construction d'hypothèses, implications, etc.), les compétences visées et les rapports entre formes et fonctions et entre morphosyntaxe et sémantique.

Notre choix n'est pas le fruit du hasard: l'œuvre de Grevisse a influencé non seulement les théorisations suivantes sur la grammaire, mais aussi la didactique de celle-ci. Pour ces raisons, s'approcher d'un texte comme Exercices sur la grammaire française et en analyser les tâches métalinguistiques nous permet de mieux comprendre la conception et le rôle de la grammaire dans la méthodologie traditionnelle, méthodologie qui a perduré pendant des siècles et qui a 
influencé non seulement l'enseignement du français langue maternelle, mais également l'enseignement/ apprentissage du français langue étrangère.

In this article we will analyse the grammar exercises included in M. Grevisse's Exercices sur la grammaire française, firstly published in Gembloux in 1942 by Duculot. We will examine and detail the different typologies of exercises (exercises of analysis, identification, transformation, creation, etc.) and their objectives (memorisation, categorisation, application, etc.), the competences required to accomplish the tasks and the relations between forms and functions and between morphology and semantics.

Our choice is not random : the works of M. Grevisse have influenced not only all of the successive grammar theorisations, but also the didactics of grammar. For those reasons, approaching a book like Exercices sur la grammaire française and analysing its metalinguistic tasks allows us to understand the role of grammar in the traditional methodology, which has continued in centuries and which has influenced not only de teaching of French as a native languages, but also the teaching/learning of French as a foreign language.

\section{INDEX}

Mots-clés : français langue étrangère, grammaire, exercices de grammaire, typologie des exercices de grammaire, méthode traditionnelle, Grevisse

Keywords : French as a foreign language, grammar, grammar exercises, typology of grammar exercises, traditional methods in language learning, Grevisse

\section{AUTEUR}

\section{ROSA LEANDRA BADALAMENTI}

Université de Palerme, Italie rosaleandra.bk@gmail.com 